www.nature.com/pj

\title{
Synthesis and microphase-separated nanostructures of P4VP-based amphiphilic liquid-crystalline block copolymer
}

\author{
Hideaki Komiyama, Hiroki Nishiyama, Jun Sawayama, Tomokazu Iyoda and Takanobu Sanji \\ An amphiphilic liquid-crystalline block copolymer composed of poly(4-vinylpyridine) (P4VP) block and polymethacrylate bearing \\ stilbene mesogens in the side chain (PMA(Stb)) was prepared by using a reversible addition-fragmentation chain-transfer \\ polymerization. Perpendicularly aligned P4VP cylindrical microdomains surrounded by PMA(Stb) matrix microdomains with a \\ liquid-crystalline smectic layer were found for the spin-coated film of the block copolymer using grazing incidence small-angle \\ $\mathrm{X}$-ray scattering and transmission electron microscopy.
}

Polymer Journal (2015) 47, 571-575; doi:10.1038/pj.2015.32; published online 20 May 2015

\section{INTRODUCTION}

Block copolymers, which consist of two chemically dissimilar polymer chains joined via a covalent bond, can self-assemble into unique macromolecular architectures, such as spheres, cylinders and lamella. ${ }^{1}$ Microphase-separated nanostructures in block copolymer thin films have attracted much attention as templates and masks for fabricating periodic nanostructures of functional materials. ${ }^{2-5}$ Recently, side-chain liquid-crystalline block copolymers have been of interest because the block copolymers are hierarchical structures composed of microphaseseparated liquid-crystal structures. Also of interest is the orientation of the microphase-separated nanostructures, which can be controlled by external fields, such as light, topological patterns of the substrate and interfacial energy, associated with the alignment of liquid-crystal moieties. ${ }^{6-9}$ In addition, side-chain liquid-crystalline block copolymers give asymmetric phase diagrams, where the cylindrical phase window is wider than that of the conventional block copolymers. ${ }^{10-12}$

We have developed a series of poly(ethylene oxide) (PEO)-based liquid-crystalline block copolymers, $\mathrm{PEO}-b$-PMA(Az), consisting of PEO and polymethacrylate with azobenzene mesogen in the side chains (PMA(Az)), using atom-transfer radical polymerization. ${ }^{13}$ This block copolymer forms hexagonally arranged and perpendicularly oriented PEO cylindrical microdomains surrounded by $\mathrm{PMA}(\mathrm{Az})$ matrix microdomains. PEO was usually employed as one of the blocks, although various liquid-crystalline mesogen units, such as azobenzene, stilbene, benzylideneaniline and chalcone, are possible in the side chain of PMA. ${ }^{14,15}$

Herein, we report the synthesis of a poly(4-vinylpyridine) (P4VP)based liquid-crystalline block copolymer, P4VP- $b$-PMA(Stb), consisting of PMA having liquid-crystal stilbene mesogen in the side chain $(\mathrm{PMA}(\mathrm{Stb}))$ as another block, using reversible addition-fragmentation chain-transfer (RAFT) polymerization. The block copolymer with P4VP as one of the blocks could be fascinating as a template for nanoscaled patterning when the block copolymer forms microphaseseparated nanostructures. This is because P4VP has the ability to bind with molecules and metals (ions) through supramolecular assembly $^{16-19}$ and coordination bonds. ${ }^{20-24}$ The block copolymer, P4VP-b-PMA(Stb), was characterized using ${ }^{1} \mathrm{H}$ NMR, gel permeation chromatography (GPC) and differential scanning calorimetry. We also investigated the microphase-separated nanostructure in the block copolymer thin film using grazing incidence small-angle $\mathrm{X}$-ray scattering (GISAXS) equipment and transmission electron microscopy (TEM).

\section{EXPERIMENTAL PROCEDURE}

General information

${ }^{1} \mathrm{H}$ NMR spectra were acquired using a Bruker Biospin AVANCE 400 FT-NMR spectrometer (Bruker Biospin GmbH, Rheinstetten, Germany) at $400 \mathrm{MHz}$. Molecular weights of polymers were determined using GPC performed on a JASCO HPLC LC-2000Plus (Jasco, Tokyo, Japan) instrument equipped with SHODEX LF804 (Shodex, Japan) $(\times 2)$ columns. The number-averaged molecular weight $\left(M_{\mathrm{n}}\right)$ and weight-averaged molecular weight $\left(M_{\mathrm{w}}\right)$ were calculated using polystyrenes (Tosoh, Tokyo, Japan) as standards.

Thermal analyses were conducted on an EXSTAR X-DSC7000 differential scanning calorimetry system (Seiko Instruments, Chiba, Japan) at a scanning rate of $\pm 10^{\circ} \mathrm{C} \mathrm{min}^{-1}$ under $\mathrm{N}_{2}$ flow. GISAXS was measured using a NanoViewer setup with a two-dimensional detector PILATUS (Rigaku, Japan). The X-ray experiment was performed using a $\mathrm{Cu} \mathrm{K \alpha}$ radiation beam $(\lambda=1.541 \AA)$, which was converged and monochromatized using a Confocal Max Flax mirror. The incidence angle of the X-rays was set to $\sim 0.2^{\circ}$ near the critical reflection angle of a Si wafer to obtain in-plane signals effectively. The samples for the measurement were prepared using a spin-coating method. A $3 \mathrm{wt} \%$ 

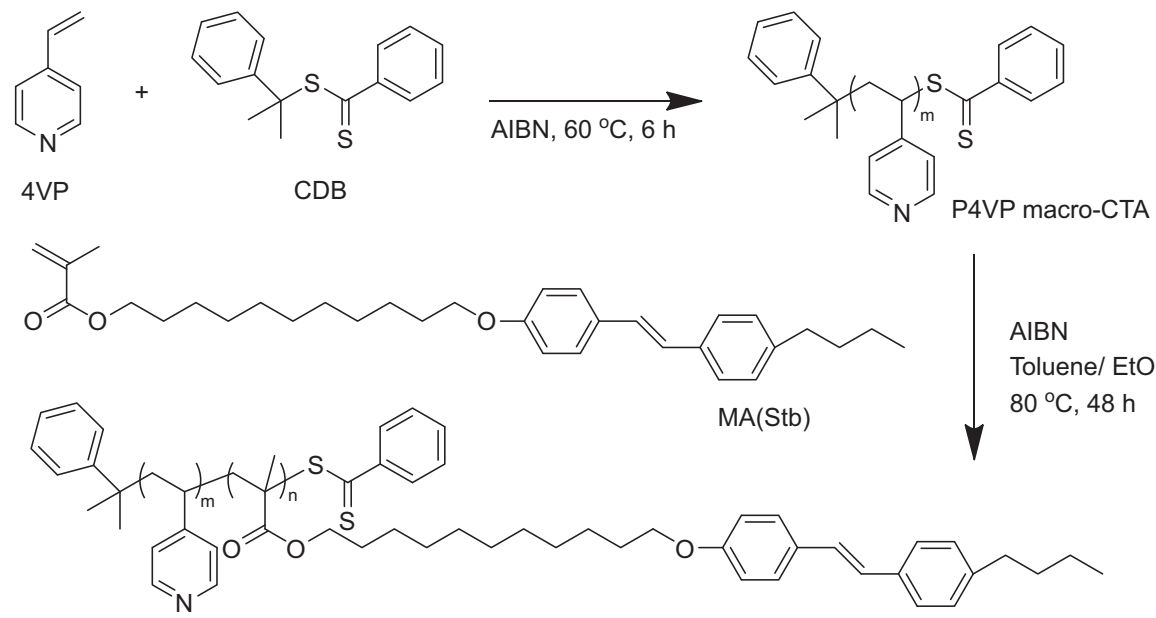

P4VP-b-PMA(Stb)

Scheme 1 Synthetic route to poly(4-vinylpyridine)- $b$-polymethacrylate with liquid-crystalline stilbene mesogens in the side chain via reversible additionfragmentation chain-transfer polymerization.
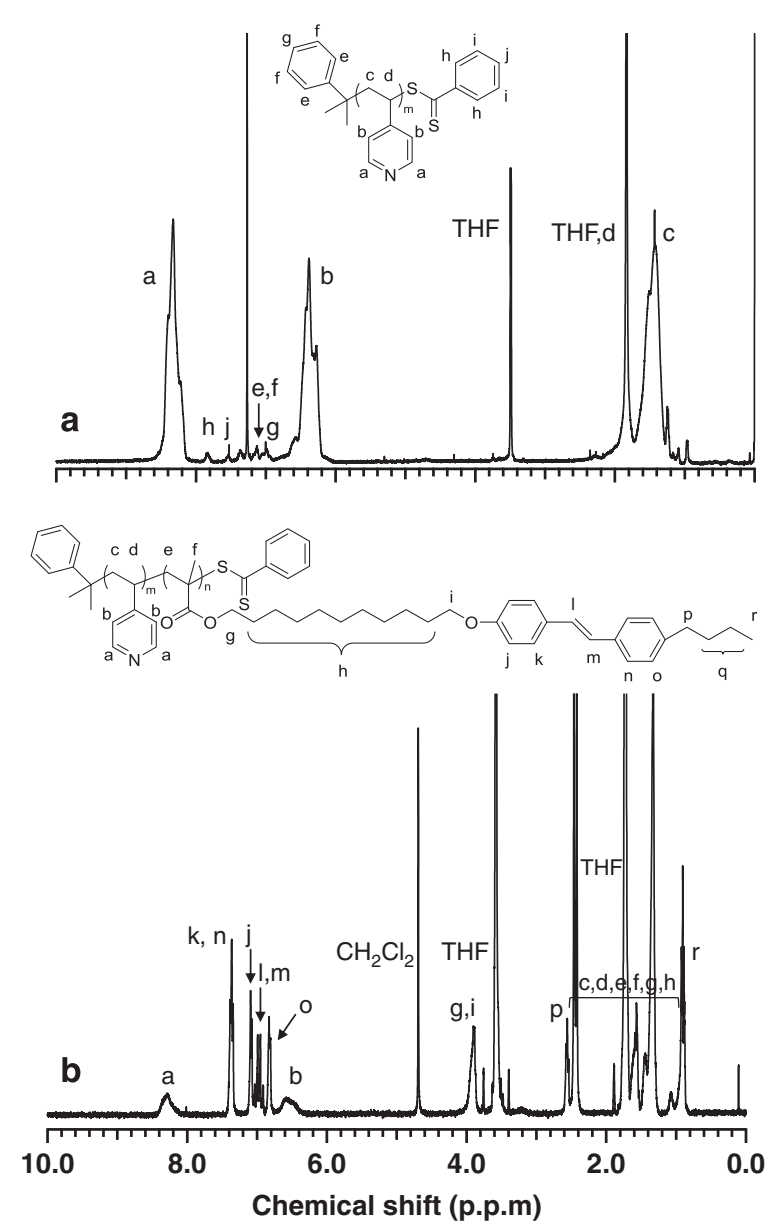

Figure $1^{1} \mathrm{H}$ NMR spectra of (a) poly(4-vinylpyridine) macro-CTA in $\mathrm{CDCl}_{3}$ and (b) poly(4-vinylpyridine)- $b$-polymethacrylate with liquid-crystalline stilbene mesogens in the side chain in THF- $d_{8}$.

solution (chloroform/THF (8/2, v/v)) of the block copolymers was spin-coated onto the $\mathrm{Si}$ wafer. Thermal annealing was carried out with the following temperature program: $190^{\circ} \mathrm{C}$ for $0.5 \mathrm{~h}$, decrease to $160^{\circ} \mathrm{C}, 160^{\circ} \mathrm{C}$ for $2 \mathrm{~h}$, decrease to room temperature without any precise control of the heating and cooling rates. TEM images were obtained using a Hitachi H-7000 electron microscope (Hitachi High-Technologies, Tokyo, Japan) at an acceleration voltage of $100 \mathrm{kV}$. The samples for observation were prepared according to the following procedure. The spin-coated thin films from a $1.5 \mathrm{wt} \%$ solution (chloroform/THF $(8 / 2, \mathrm{v} / \mathrm{v})$ ) onto a fresh surface of mica substrate were thermally annealed under vacuum. The resulting thin films were easily peeled from the substrates by soaking in water, then transferred onto copper TEM grids, and exposed in $\mathrm{RuO}_{4}$ vapor at room temperature for $3 \mathrm{~min}$ to stain the P4VP microdomains.

\section{Materials}

Cumyl dithiobenzoate and 4-vinylpyridine were purchased from Aldrich. Azobisisobutyronitrile was recrystallized from ethanol before use. The monomer 11-(4-((E)-4-butylstyryl)phenoxy)undecyl methacrylate (MA(Stb)) was synthesized according to our previous report. ${ }^{14,25}$

\section{Preparation of P4VP Macro-CTA}

RAFT polymerization was used to prepare P4VP macro-chain-transfer agent (P4VP macro-CTA) (Scheme 1). P4VP macro-CTA was synthesized under bulk conditions by following the reported literature. ${ }^{26}$ An amount of $13.3 \mathrm{mg}$ $(5.47 \mathrm{mmol})$ of azobisisobutyronitrile and $103.7 \mathrm{mg}$ (25.6 mmol) of cumyl dithiobenzoate were mixed in $14.8 \mathrm{ml}$ of 4-vinylpyridine. The polymerization was performed in bulk conditions under an argon atmosphere at $60^{\circ} \mathrm{C}$ for $6 \mathrm{~h}$. After adding a small amount of THF, the polymer was precipitated in toluene, filtered, and finally dried under vacuum at room temperature. P4VP macroCTA was obtained as a pink powder $(1.19 \mathrm{~g}, 13 \%)$. The number-average degree of polymerization (DP) and $M_{\mathrm{n}}$ (NMR) were estimated from ${ }^{1} \mathrm{H}$ NMR measurements in $\mathrm{CDCl}_{3}$ to be 77 and 8100 , respectively. $M_{\mathrm{n}}(\mathrm{GPC})$ and $M_{\mathrm{w}} /$ $M_{\mathrm{n}}$ were obtained from a GPC measurement using $\mathrm{N}, \mathrm{N}$-dimethylformamide (DMF) solution with $10-\mathrm{mm} \mathrm{LiCl}$ as an elution solution and found to be 7800 and 1.25 , respectively.

\section{Preparation of P4VP-b-PMA(Stb)}

Amounts of $0.101 \mathrm{~g}(0.0139 \mathrm{mmol})$ of P4VP, $5.7 \mathrm{mg}(0.00348 \mathrm{mmol})$ of azobisisobutyronitrile, and $685.5 \mathrm{mg}(1.39 \mathrm{mmol})$ of $\mathrm{MA}(\mathrm{Stb})$ were mixed in a test tube, degassed, and filled with argon. Five milliliters of ethanol and $8 \mathrm{ml}$ of toluene were added using a syringe. After argon bubbling, the mixture was heated at $80^{\circ} \mathrm{C}$ for $24 \mathrm{~h}$. After the solvent was removed under reduced pressure, the residual solid was dissolved in a minimum amount of dichloromethane, and then reprecipitated in hot hexane repeatedly to remove the remaining MA (Stb) monomer. After filtering and drying, P4VP- $b$-PMA(Stb) was obtained as a light-pink solid (530.6 mg, 67\%). 


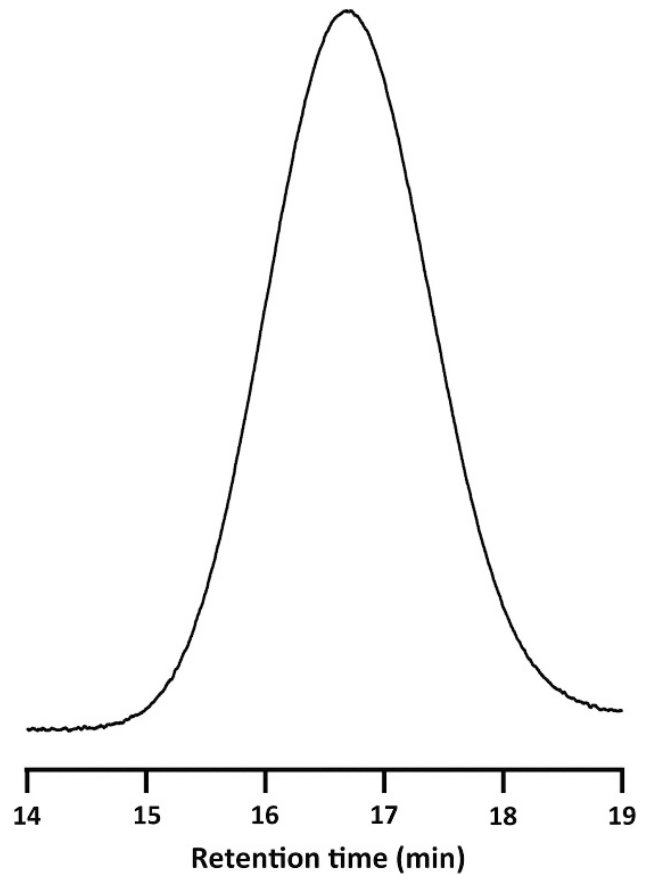

Figure 2 Gel permeation chromatography curve of poly(4-vinylpyridine)- $b$ polymethacrylate with liquid-crystalline stilbene mesogens in the side chain.

\section{RESULTS AND DISCUSSION}

Block copolymerization using RAFT polymerization

RAFT polymerization is a powerful polymerization technique for the preparation of block copolymers from a wide variety of monomers..$^{27,28}$ In this study, a P4VP macro-CTA was used for the polymerization of the $\mathrm{MA}(\mathrm{Stb})$ monomer. The P4VP macro-CTA was prepared by the polymerization of 4 -vinylpyridine with cumyl dithiobenzoate. ${ }^{26}$ GPC showed the molecular weight and the polydispersity index of the macro initiator to be 7800 and 1.25, respectively. Figure la shows the ${ }^{1} \mathrm{H}$ NMR spectrum of P4VP macro-CTA in $\mathrm{CDCl}_{3}$. DP for the PMA(Stb) segment and the number average molecular weight $\left(M_{\mathrm{n}}(\mathrm{NMR})\right)$ estimated from the spectrum were 77 and 8100 , respectively. P4VP macro-CTA was then conducted on the RAFT polymerization of MA(Stb) (Scheme 1). After polymerization, the polymer was obtained in $67 \%$ yield. Figure $1 \mathrm{~b}$ shows the ${ }^{1} \mathrm{H}$ NMR spectrum of P4VP- $b-\mathrm{PMA}(\mathrm{Stb})$ in THF- $d_{8}$. The peak assignments are also shown in the figure. DP for the PMA(Stb) segment and $M_{\mathrm{n}}(\mathrm{NMR})$ of P4VP- $b$-PMA(Stb) were 77 and 45800 , respectively, calculated from the integral intensity ratio of the resonances at 8.3 p.p.m. of the $\mathrm{H}_{\mathrm{a}}$ protons of the P4VP segment and 3.9 p.p.m. for the $\mathrm{H}_{\mathrm{g}, \mathrm{i}}$ protons in the side chain of the $\mathrm{PMA}(\mathrm{Stb})$ segment. The weight fraction of the P4VP block was 0.18. Figure 2 shows the GPC curve of P4VP- $b$-PMA(Stb). The $M_{\mathrm{w}} / M_{\mathrm{n}}$ value is low (1.59), indicating that the RAFT polymerization was reasonably controlled. $M_{\mathrm{n}}(\mathrm{GPC})$ (24600) was lower than $M_{\mathrm{n}}(\mathrm{NMR})$ (45800), probably because of adsorption of the P4VP block to the GPC columns.

\section{Microphase-separated nanostructure in a thin film}

The thermal properties of a solid thin film of P4VP- $b$-PMA(Stb) were investigated using differential scanning calorimetry. Figure 3 displays the differential scanning calorimetry curves of P4VP macro-CTA and P4VP$b$-PMA(Stb) in the first cooling and second heating processes. The $\mathrm{P} 4 \mathrm{VP}$ macro-CTA showed a glass transition at $140{ }^{\circ} \mathrm{C}$ in the heating process (Figure 3a). In the case of P4VP- $b$-PMA(Stb), two transition peaks were clearly found at 150 and $170{ }^{\circ} \mathrm{C}$ in the cooling and heating processes
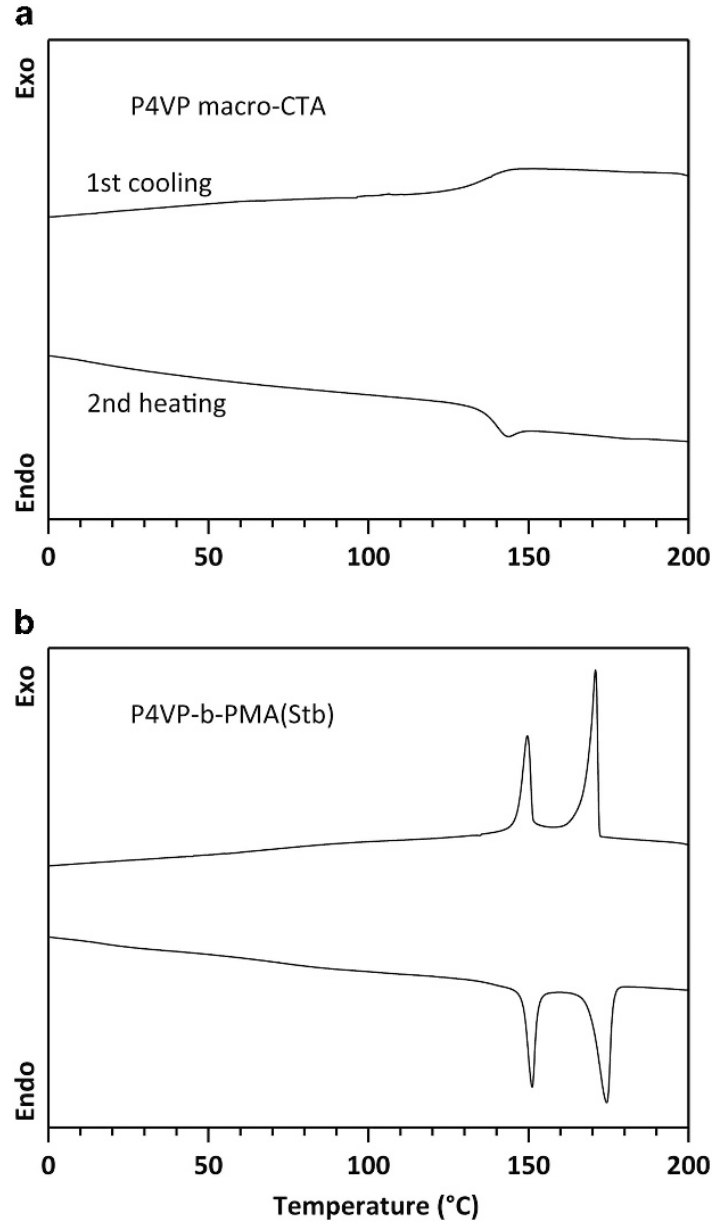

Figure 3 Differential scanning calorimetry curves of (a) poly(4-vinylpyridine) macro-chain-transfer agent and (b) poly(4-vinylpyridine)- $b$-polymethacrylate with liquid-crystalline stilbene mesogens in the side chain on the first cooling and second heating processes with heating/cooling rates of $10^{\circ}$ $\mathrm{C}$ min $^{-1}$.

(Figure $3 \mathrm{~b}$ ). In the cooling process, the peak at higher temperature was assigned to a phase transition from an isotropic phase to smectic A (SmA) of the liquid-crystalline stilbene moiety in the side chain of PMA, which is in good agreement with that reported previously. ${ }^{14}$ The peak at lower temperature was identified as a phase transition between SmA and hexatic-type phases. In the heating process, the glass transition of P4VP macro-CTA was not observed, probably because of the short P4VP block in P4VP-b-PMA(Stb) (weight fraction of P4VP block, 0.18). Based on this observation, the thermal annealing processes were determined using the following temperature program: $190^{\circ} \mathrm{C}$ for $0.5 \mathrm{~h}$, decrease to $160^{\circ} \mathrm{C}$, $160^{\circ} \mathrm{C}$ for $2 \mathrm{~h}$, decrease to room temperature without any precise control of the heating and cooling rates.

The thin film of P4VP- $b$-PMA(Stb) with a thickness of $300 \mathrm{~nm}$ on the $\mathrm{Si}$ wafer was prepared using a spin-coating method, and the GISAXS measurement was examined to characterize the microphaseseparated nanostructures. Before the GISAXS measurement, we investigated the surface uniformity of the film with AFM. The value of root mean square was $0.98 \mathrm{~nm}$, showing the smooth surface. Figure 4 shows the GISAXS profiles of the P4VP- $b$-PMA(Stb) thin film. In the GISAXS two-dimensional image, two peaks appeared in an out-of-plane region, while a couple of peaks appeared in an in-plane region (Figure $4 \mathrm{a}$ ). Figure $4 \mathrm{~b}$ shows the out-of-plane intensity profile 


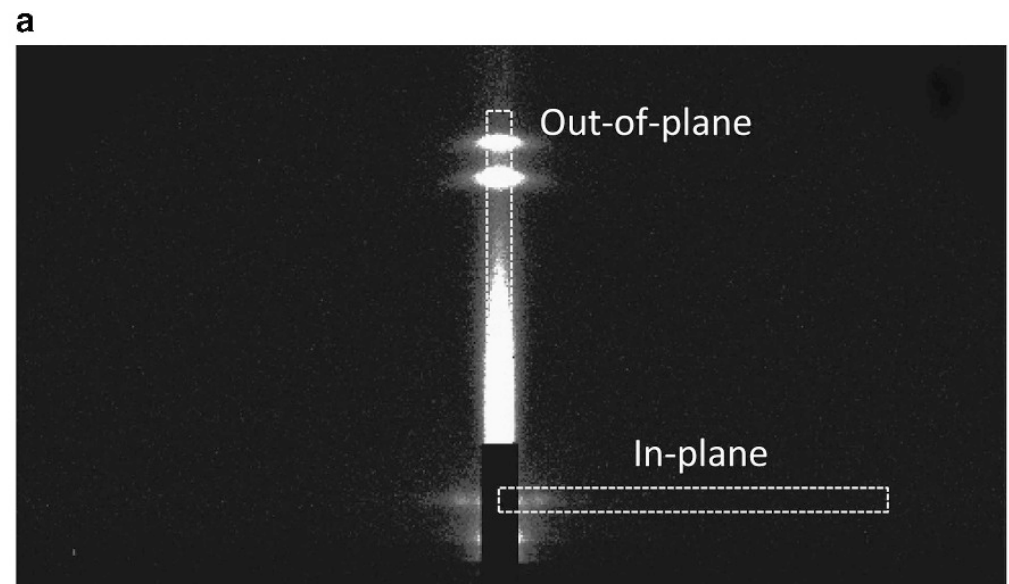

b

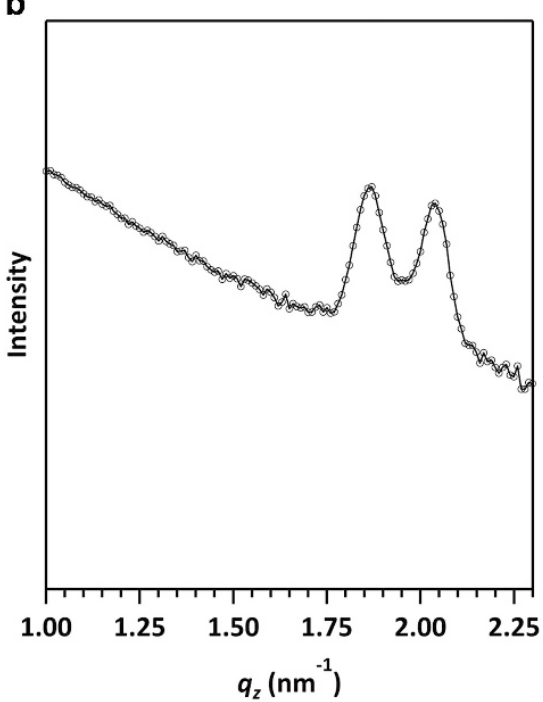

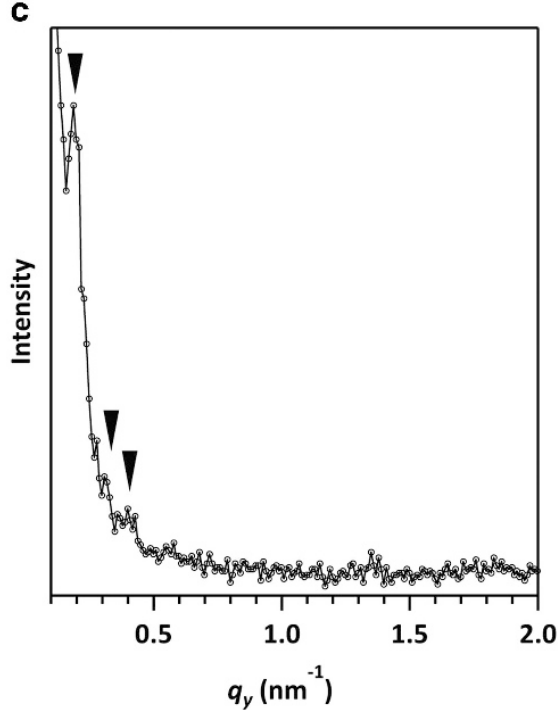

Figure 4 (a) Grazing incidence small-angle X-ray scattering two-dimensional image of poly(4-vinylpyridine)-b-polymethacrylate with liquid-crystalline stilbene mesogens in the side chain film on Si wafer. (b) Out-of-plane and (c) in-plane profiles of the grazing incidence small-angle X-ray scattering twodimensional image.

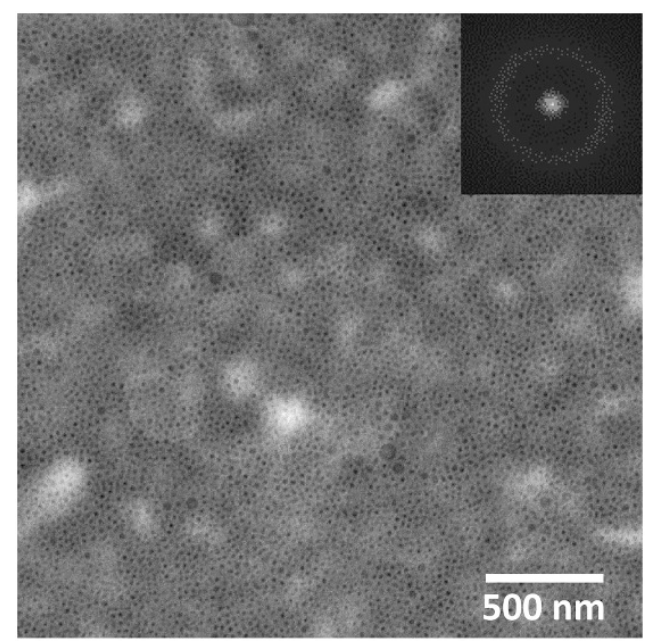

Figure 5 Transmission electron microscope image of poly(4-vinylpyridine)- $b$ polymethacrylate with liquid-crystalline stilbene mesogens in the side chain thin film stained with $\mathrm{RuO}_{4}$. Inset is the corresponding fast Fourier transform image. of the corresponding two-dimensional image, indicating the liquidcrystalline stilbene layers with smectic A phase. The lower and higher scattering peaks in the $q_{z}$-vector originate from scattering of the transmitted and reflected X-ray beams on the Si wafer, respectively. ${ }^{29}$ The periodicity of the smectic A layers was calculated from these peaks to be $3.67 \mathrm{~nm}$, which is consistent with the reported periodicity in PEO- $b$-PMA(Stb) thin film. ${ }^{14}$ No peaks assigned to the stilbene layer

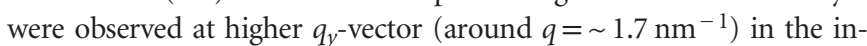
plane direction (Figure $4 \mathrm{c}$ ). These results indicate that homeotropic alignment of the stilbene layers. In the in-plane profile, the scattering peaks were $0.188,0.318$ and $0.378 \mathrm{~nm}^{-1}$ in the lower $q_{y}$-vector, corresponding to $1, \sqrt{3}$ and $\sqrt{ } 4$ as the (10), (11) and (20) planes of the hexagonally arranged cylindrical structure, respectively. The P4VP segment formed cylindrical microdomains in the $\mathrm{PMA}(\mathrm{Stb})$ matrix microdomains with a perpendicular orientation to the Si wafer, based on the composition ratio between the P4VP and PMA(Stb) segments (the weight fraction of the P4VP segment, 0.18). The center-to-center distance, estimated from the first peak position, was $38.6 \mathrm{~nm}$.

The TEM image of the P4VP- $b$-PMA(Stb) thin film with $<100 \mathrm{~nm}$ thickness after staining with $\mathrm{RuO}_{4}$ shows microphase-separated P4VP cylindrical microdomains (Figure 5). The P4VP microdomains 
selectively stained with $\mathrm{RuO}_{4}$ partly exhibit as hexagonally arranged dots with darker contrast. No line pattern was resolved in the TEM image, indicating that the P4VP cylindrical microdomains were perpendicularly aligned to the substrate. The GISAXS also indicated a hexagonal arrangement for the P4VP cylindrical microdomains macroscopically. The center-to-center distance was calculated from the fast Fourier transform image of the TEM image to be $38 \mathrm{~nm}$, in good agreement with the value obtained from the GISAXS measurement.

In conclusion, the P4VP-based amphiphilic liquid-crystalline block copolymer with functional stilbene mesogens in the side chains of the PMA segment was synthesized using RAFT polymerization via P4VP macro-CTA. The thermal analysis showed the two phase transitions to the smectic A and hexatic-type phases. The macroscopic and microscopic observations by GISAXS and TEM showed that the P4VP cylindrical microdomains were perpendicularly oriented to the substrate after thermal annealing. The thin film of the block copolymer with the ability to metal-bind in the P4VP block is expected to act as a template to fabricate periodic arrays of metal nanostructures. Further work along this line is in progress.

1 Bates, F. S,. \& Fredrickson, G. H. Block copolymers-designer soft materials. Physics Today. 52, 32-38 (1999).

2 Park, M., Harrison, C., Chaikin, P. M., Register, R. A. \& Adamson, D. H. Block copolymer lithography: periodic arrays of $\sim 10^{11}$ holes in 1 square centimeter. Science 276, 1401-1404 (1997).

3 Thurn-Albrecht, T., Schotter, J., Kästle, G. A., Emley, N., Shibauchi, T., Krusin-Elbaum, L., Guarini, K., Black, C. T., Tuominen, M. T. \& Russell, T. P. Ultrahigh-density nanowire arrays grown in self-assembled diblock copolymer templates. Science 290, 2126-2129 (2000)

4 Lee, J. I., Cho, S. H., Park, S.-M., Kim, J. K., Kim, J. K., Yu, J. W., Kim, Y. C. \& Russell, T. P. Highly aligned ultrahigh density arrays of conducting polymer nanorods using block copolymer templates. Nano Lett. 8, 2315-2320 (2008).

5 Kamcev, J., Germack, D. S., Nykypanchuk, D., Grubbs, R. B., Nam, C. Y. \& Black, C. T. Chemically enhancing block copolymers for block-selective synthesis of self-assembled metal oxide nanostructures. ACS Nano 7, 339-346 (2013).

$6 \mathrm{Yu}, \mathrm{H}$., lyoda, T. \& Ikeda, T. Photoinduced alignment of nanocylinders by supramolecular cooperative motions. J. Am. Chem. Soc. 128, 11010-11011 (2006).

7 Fukuhara, K., Fujii, Y., Nagashima, Y., Hara, M., Nagano, S. \& Seki, T. Liquid crystalline polymer and block copolymer domain alignment controlled by free-surface segregation. Angew. Chem. Int. Ed. 38, 5749-5751 (2013).

8 Sano, M., Nakamura, S., Hara, M., Nagano, S., Shinohara, Y., Amemiya, Y. \& Seki, T. Pathways toward photoinduced alignment switching in liquid crystalline block copolymer films. Macromolecules 47, 7178-7186 (2014).

9 Komura, M., Yoshitake, A., Komiyama, H. \& Iyoda, T. Control of air-interface-induced perpendicular nanocylinder orientation in liquid crystal block copolymer films by a surface-covering method. Macromolecules 48, 672-678 (2015).
10 Anthamatten, M., Zhen, W. Y. \& Hammond, P. T. A morphological study of well-defined smectic side-chain Lc block copolymers. Macromolecules 32, 4838-4848 (1999).

11 Anthamatten, M. \& Hammond, P. T. Free-energy model of asymmetry in side-chain liquid-crystalline diblock copolymers. J. Polym. Sci. B Polym.Phys 39, 2671-2691 (2001).

12 Komiyama, H., Sakai, R., Hadano, S., Asaoka, S., Kamata, K., lyoda, T., Komura, M., Yamada, T. \& Yoshida, H. Enormously wide range cylinder phase of liquid crystalline PEO-b-PMA(Az) block copolymer. Macromolecules 47, 1777-1782 (2014).

13 Tian, Y., Watanabe, K., Kong, X., Abe, J. \& Iyoda, T. Synthesis, nanostructures, and functionality of amphiphilic liquid crystalline block copolymers with azobenzene moieties. Macromolecules 35, 3739-3747 (2002).

14 Asaoka, S., Uekusa, T., Tokimori, H., Komura, M., Iyoda, T., Yamada, T. \& Yoshida, H. Normally oriented cylindrical nanostructures in amphiphilic PEO-LC diblock copolymers films. Macromolecules 44, 7645-7658 (2011).

15 Miyatake, M., Kimura, T., Komiyama, H., Komura, M. \& Iyoda, T. Large-area fabrication of free-standing thick membrane with microphase-separated cylindrical nanostructure. Trans. Mater. Res. Soc. Japan 37, 409-412 (2012).

16 Sidorenko, A., Tokarev, I., Minko, S. \& Stamm, M. Ordered reactive nanomembranes/ nanotemplates from thin films of block copolymer supramolecular assembly. J. Am. Chem. Soc. 125, 12211-12216 (2003).

17 Tang, C., Lennon, E. M., Fredrickson, G. H., Kramer, E. J. \& Hawker, C. J. Evolution of block copolymer lithography to highly ordered square arrays. Science 322, 429-432 (2008).

18 Kuila, B. K., Nandan, B., hme, M. B., Janke, A. \& Stamm, M. Vertically oriented arrays of polyaniline nanorods and their super electrochemical properties. Chem. Commun. 5749-5751 (2009).

19 Kuila, B. K. \& Stamm, M. Fabrication of oriented polyaniline nanostructures using block copolymer nanotemplates and their optical, electrochemical and electric properties. J. Mater. Chem. 20, 6086-6094 (2010).

20 Park, S., Wang, J. Y., Kim, B., Xu, J. \& Russell, T. P. A simple route to highly oriented and ordered nanoporous block copolymer templates. ACS Nano 2, 766-772 (2008).

21 Cho, W. J., Kim, Y. \& Kim, J. K. Ultrahigh-density array of silver nanoclusters for sers substrate with high sensitivity and excellent reproducibility. ACS Nano 6, 249-255 (2012).

22 Aizawa, M. \& Buriak, J. M. Block copolymer-templated chemistry on Si, Ge, Inp and Gaas Surfaces. J. Am. Chem. Soc. 127, 8932-8933 (2005).

23 Aizawa, M. \& Buriak, J. M. Nanoscale patterning of two metals on silicon surfaces using an ABC triblock copolymer template. J. Am. Chem. Soc. 128, 5877-5886 (2006).

24 Aizawa, M. \& Buriak, J. M. Block copolymer templated chemistry for the formation of metallic nanoparticle arrays on semiconductor surfaces. Chem. Mater. 19, 5090-5101 (2007)

25 Yamamoto, T., Kimura, T., Komura, M., Suzuki, Y., Iyoda, T., Asaoka, S. \& Nakanishi, $\mathrm{H}$. block copolymer permeable membrane with visualized high-density straight channels of poly(ethylene oxide). Adv. Funct. Mater. 21, 918-926 (2011).

26 Convertine, A. J., Sumerlin, B. S., Thomas, D. B., Lowe, A. B. \& McCormick, C. L. Synthesis of block copolymers of 2- and 4-vinylpyridine by RAFT polymerization. Macromolecules 36, 4679-4681 (2003).

27 Chiefari, J., Chong, Y. K. B., Ercole, F., Krstina, J., Jeffery, J., Le, T. P. T., Mayadunne, R. T. A., Meijs, G. F., Moad, C. L., Moad, G., Rizzardo, E. \& Thang, S. $\mathrm{H}$. Living free-radical Polymerization by Reversible Addition-Fragmentation Chain Transfer: The RAFT Process. Macromolecules 31, 5559-5562 (1998).

28 Mccormick, C. L. \& Lowe, A. B. Aqueous RAFT Polymerization: Recent Developments in Synthesis of Functional Water-Soluble (Co)polymers with Controlled Structures. Acc. Chem. Res. 37, 312-325 (2004).

29 Komura, M., Watanabe, K., Iyoda, T., Yamada, T., Yoshida, H. \& Iwasaki, Y. Laboratory-GISAXS measurements of block copolymer films with highly ordered and normally oriented nanocylinders. Chem. Lett. 38, 408-409 (2009). 